\title{
Strong cosmic censorship for a scalar field in a Born-Infeld-de Sitter black hole
}

\author{
Qingyu Gan, ${ }^{*}$ Guangzhou Guo๑, ${ }^{\dagger}$ Peng Wang, ${ }^{\ddagger}$ and Houwen $\mathrm{Wu}^{\S}$ \\ Center for Theoretical Physics, College of Physics, Sichuan University, Chengdu 610064, \\ People's Republic of China
}

(Received 16 August 2019; published 2 December 2019)

\begin{abstract}
It has been shown that the strong cosmic censorship (SCC) can be violated by a scalar field in a nearextremal Reissner-Nordstrom-de Sitter black hole. In this paper, we investigate the strong cosmic censorship in a Born-Infeld-de Sitter black hole by a scalar perturbation field with/without a charge. When the Born-Infeld parameter $b$ becomes small, the nonlinear electrodynamics effect starts to play an important role and tends to rescue SCC. Specifically, we find that the SCC violation region decreases in size with decreasing $b$. Moreover, for a sufficiently small $b$, SCC can always be restored in a near-extremal BornInfeld-de Sitter black hole with a fixed charge ratio.
\end{abstract}

DOI: 10.1103/PhysRevD.100.124009

\section{INTRODUCTION}

A black hole formed from gravitational collapse could possess a curvature singularity. If a timelike singularity is formed, the undetermined initial data on it would cause the breakdown of determinism of general relativity. On the other hand, it is well known that there exist some solutions of the Einstein field equations admitting the presence of timelike singularities, e.g., Kerr-Newman black holes, Reissner-Nordstrom black holes. To rescue the predictability of general relativity, Penrose proposed a conjecture, namely the strong cosmic censorship (SCC), asserting that the maximal Cauchy development of physically acceptable initial conditions is locally inextendible as a regular manifold [1-3]. Consequently, when the initial data are perturbed outside of a black hole, whether SCC holds true crucially depends on the extendibility of the perturbation (e.g., the metric and other fields) at the Cauchy horizon, which encloses the timelike singularity.

Nevertheless, there are some subtleties of describing the extension of the perturbation at the Cauchy horizon, and hence several formulation versions of SCC have been proposed. For example, the perturbation cannot be

\footnotetext{
*qygan@stu.scu.edu.cn

tyangzhouguo@stu.scu.edu.cn

pengw@scu.edu.cn

\$iverwu@scu.edu.cn
}

Published by the American Physical Society under the terms of the Creative Commons Attribution 4.0 International license. Further distribution of this work must maintain attribution to the author(s) and the published article's title, journal citation, and DOI. Funded by SCOAP ${ }^{3}$.
$C^{r}(r \in N)$ smooth at the Cauchy horizon in the $C^{r}$ version of SCC $[4,5]$. However, since weak solutions can have many important physical applications in which $C^{r}$ smooth solutions are not available, a more appropriate way to characterize the extendibility is whether perturbation being inextendible as a weak solution of the equations of motion. Specifically, a weak solution of the Einstein equation is characterized by locally square integrable Christoffel symbols in some charts of the spacetime manifold. This observation then leads to the Christodoulou version of SCC, which states that the metric cannot be extendible across the Cauchy horizon with locally square integrable Christoffel symbols [6]. In a physical intuitive way, an observer generically cannot cross the Cauchy horizon in the manner of the classical equation of motion. For a toy model with a linear scalar field perturbation on fixed background, the Christodoulou formulation of SCC asserts that the scalar field perturbation will not belong to the Sobolev space $H_{\mathrm{loc}}^{1}$ at the Cauchy horizon. In other words, if SCC is violated in the Christodoulou version, the perturbation belongs to $H_{\mathrm{loc}}^{1}$ and, roughly speaking, has finite energy at the Cauchy horizon. Note that if the perturbation belongs to the Sobolev space $H_{\text {loc }}^{1}$, its first derivative is locally square integrable.

The possible singular behavior of a perturbation at the Cauchy horizon comes from the mass-inflation mechanism, associated with the exponential amplification due to the blue shift effect [7-12]. However, there is another mechanism competing with the mass-inflation mechanism to invalidate SCC: the time-dependent remnant perturbation decaying outside of the black hole. When the perturbation decays slowly enough, SCC could be valid. In fact, a perturbation in an asymptotically flat black hole satisfies an inverse power law decay [13-15], which ensures 
the mass-inflation mechanism is strong enough to render the Cauchy horizon unstable upon perturbation $[8,16]$. On the other hand, it was observed that a remnant perturbation can exponentially decay in a black hole in asymptotically de Sitter (dS) spacetime [17-24], which implies that the perturbation might have chance to decay fast enough to violate SCC. More precisely, it showed that, for an asymptotically dS black hole, the competition between the mass inflation and remnant decaying can be characterized by [25-30]

$$
\beta \equiv \frac{\alpha}{\kappa_{-}},
$$

where $\kappa_{-}$denotes the surface gravity at the Cauchy horizon. Here, $\alpha$ is the spectral gap representing the distance from the real axis to the lowest-lying quasinormal mode (QNM) on the lower half complex plane of frequency, namely $\alpha \equiv$ $\inf _{\ln }\left\{-\operatorname{Im}\left(\omega_{\ln }\right)\right\}$ for a series of QNMs $\omega_{\ln }$. Note that $\beta>$ $1 / 2$ can lead to a potential violation of the Christodoulou version of SCC.

Recently, the validity of the Christodoulou version of $\mathrm{SCC}$ has been explored in asymptotically dS black holes by computing $\beta$ for various perturbation fields [30-43]. In particular, a massless neutral scalar perturbation field in a Reissner-Nordstrom-de Sitter (RN-dS) black hole was considered in [30], and it was proven that SCC is violated in the near-extremal regime. Since the charge matter is necessary for the formation of a charged black hole by gravitational collapse, the analysis was then extended to a charged scalar field in a RN-dS black hole [31-33], which showed that, in the highly extremal limit, there always exists a region in parameter space where SCC is violated. Although it was claimed in [34] that SCC would be saved for sufficiently large scalar field mass and charge, the existence of arbitrarily small oscillations of $\beta$ around $\beta=$ $1 / 2$ was observed in a sufficiently near-extremal black hole [32]. These oscillations were dubbed as "wiggles," which result from nonperturbative effects and can lead to a violation of SCC for an arbitrary large scalar field charge. Later, SCC in a RN-dS black hole was also discussed in the context of the Dirac perturbation field $[35,36]$ and higher spacetime dimensions [37,38], where there still exists some room for the violation of SCC. Considering smooth initial data, the violation of SCC becomes more severe for the coupled linearized electromagnetic and gravitational perturbations in a RN-dS black hole [39]. In [40], the authors proved that nonlinear effects could not save SCC from being violated for a near-extremal RN-dS black hole. On the other hand, SCC is always respected for the massless scalar field and linearized gravitational perturbations in a Kerr-dS black hole [37,41].

Taking quantum contributions into account, nonlinear corrections are usually added to the Maxwell Lagrangian, which gives the nonlinear electrodynamics (NLED). Among various NLED, Born-Infeld (BI) electrodynamics, which was first introduced to smooth divergences of the electrostatic self-energy of point charges, has attracted considerable attention in the literature. Furthermore, BI electrodynamics can come from the low-energy limit of string theory and encodes the low-energy dynamics of D-branes [44]. The BI black hole solution in anti-de Sitter space was first obtained in $[45,46]$. Since then, various aspects of BI black holes have been extensively investigated, e.g., the thermodynamics and phase structure [47-52], the holographic models [53-60]. Specifically, the weak cosmic censorship (WCC) has recently been studied in a BI black hole $[61,62]$, where it was found that there may exist some counterexamples to WCC.

Until now, the charge sector of testing the Christodoulou version of SCC has been confined to Maxwell's theory of electrodynamics. Little is known about the NLED effect on the validity of SCC. In this paper, we investigate the Christodoulou version of SCC for a scalar perturbation field propagating in a BI-dS black hole. Our results show that the NLED effect tends to alleviate the violation of SCC in the near-extremal regime. Especially, for a near-extremal BI-dS black hole with a fixed charge ratio, SCC can always be saved as long as the NLED effect is strong enough. Furthermore, the parameter region where SCC is violated decreases in size when the NLED effect becomes stronger.

The rest of the paper is organized as follows. In Sec. II, we briefly review the BI-dS black hole solution and obtain the parameter region where the Cauchy horizon exists. In Sec. III, we show how to compute the QNMs for a charged and massive scalar field in a BI-dS black hole. In Sec. IV, we present and discuss the numerical results in various parameter regions. We summarize our results in the last section. For simplicity, we set $16 \pi G=c=1$ in this paper.

\section{BI-dS BLACK HOLE}

In this section, we first review the BI-dS black hole solution and then give the "allowed" region in the parameter space, in which the Cauchy horizon exists. Consider a $(3+1)$-dimensional Einstein-Born-Infeld action in the presence of the positive cosmological constant $\Lambda$, which is given by

$$
S=\int d^{4} x \sqrt{-g}\left[\mathcal{R}-2 \Lambda+4 b^{2}\left(1-\sqrt{1+\frac{F^{\mu \nu} F_{\mu \nu}}{2 b^{2}}}\right)\right],
$$

where $\mathcal{R}$ is the Ricci scalar curvature, $F_{\mu \nu}=\partial_{\mu} A_{\nu}-\partial_{\nu} A_{\mu}$ is the electromagnetic tensor field of a $\mathrm{BI}$ electromagnetic field $A_{\mu}$, and the Born-Infeld parameter $b$ is related to the string tension $\alpha^{\prime}$ as $b=1 /\left(2 \pi \alpha^{\prime}\right)$ [44]. It is noteworthy that BI electrodynamics would reduce to Maxwell electrodynamics in the limit of $b \rightarrow \infty$. So, the NLED effect in BI electrodynamics will become stronger for a smaller value of $b$. For the action (2.1), a static spherically symmetric black hole solution was obtained in $[45,46]$, 


$$
\begin{aligned}
d s^{2} & =-f(r) d t^{2}+\frac{d r^{2}}{f(r)}+r^{2}\left(d \theta^{2}+\sin ^{2} \theta d \varphi^{2}\right), \\
\mathbf{A} & =A_{t} d t=-\frac{Q}{r}{ }_{2} \mathcal{F}_{1}\left[\frac{1}{4}, \frac{1}{2}, \frac{5}{4},-\frac{Q^{2}}{b^{2} r^{4}}\right] d t,
\end{aligned}
$$

with the blackening factor

$$
\begin{aligned}
f(r)= & 1-\frac{\Lambda r^{2}}{3}-\frac{M}{8 \pi r}+\frac{2 b^{2} r^{2}}{3}\left(1-\sqrt{1+\frac{Q^{2}}{b^{2} r^{4}}}\right) \\
& +\frac{4 Q^{2}}{3 r^{2}}{ }_{2} \mathcal{F}_{1}\left[\frac{1}{4}, \frac{1}{2}, \frac{5}{4},-\frac{Q^{2}}{b^{2} r^{4}}\right] .
\end{aligned}
$$

Here, ${ }_{2} \mathcal{F}_{1}$ is the hypergeometric function, and $M$ and $Q$ are the mass and electric charge of the BI-dS black hole, respectively. Note that though the hypergeometric function ${ }_{2} \mathcal{F}_{1}[a, b, c, z]$ has a convergent series expansion for $|z|<1$, it has an analytic continuation in the complex plane with branch points at $z=1$ and $z=\infty$. Specifically, when $\operatorname{Re}(a)>0$ and $\operatorname{Re}(b)>0$, there exists an integral representation for ${ }_{2} \mathcal{F}_{1}[a, b, c, z]$ in the complex plane except for a cut along the real axis from $z=1$ to $z=\infty$. Since $z=-\frac{Q^{2}}{b^{2} r^{4}}<0$ in Eqs. (2.2) and (2.3), the hypergeometric function in these equations is well defined.

A BI-dS black hole can possess one, two, or three horizons depending on the parameters $M, Q, \Lambda$, and $b$. The topology of BI-dS black holes has been discussed in $[63,64]$. In this paper, we investigate SCC and hence focus on the BI-dS black holes possessing three horizons, namely the Cauchy horizon $r_{-}$, the event horizon $r_{+}$, and the cosmological horizon $r_{c}$. To determine the number of roots of $f(r)$, we instead consider $r f(r)$, which has the same positive roots as $f(r)$, and find that

$$
\frac{d(r f(r))}{d r}=1+\left(2 b^{2}-\Lambda\right) r^{2}-2 b \sqrt{Q^{2}+b^{2} r^{4}} .
$$

In order for $r f(r)$ to have three positive roots, the parameters must satisfy the following conditions:

(i) $0<\Lambda<2 b^{2}$ and $b Q>\frac{1}{2}$ : Since the BI-dS black hole solution is asymptotically dS, one has $r f(r) \rightarrow$ $-\infty$ in $r \rightarrow \infty$. Thus, $r f(r)$ must have a local minimum at $r=r_{\min }$ and a local maximum at $r=r_{\max }$, which has $0<r_{\min }<r_{\max }$. The existence of two extrema for $r f(r)$ requires that $d(r f(r)) /$ $d r=0$ has two positive roots, which gives $0<\Lambda<$ $2 b^{2}$ and $b Q>\frac{1}{2}$.

(ii) $M<\Delta(Q)$ : At $r=0, r f(r)$ must be greater than zero. As $r \rightarrow 0$, one has

$$
r f(r)=\frac{\Delta(Q)-M}{8 \pi}-\left(\frac{10}{3} b Q-1\right) r+\mathcal{O}\left(r^{2}\right),
$$

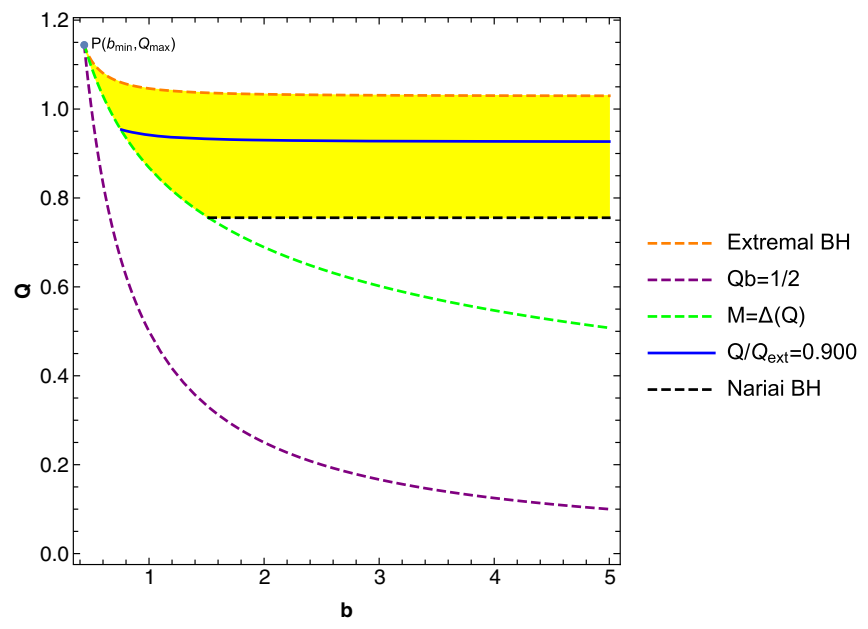

FIG. 1. $M=16 \pi$ and $\Lambda=0.14$. The BI-dS black hole in the yellow region possesses three horizons. The dashed orange and black lines represent the extremal black hole with $r_{-}=r_{+}$and the Nariai black hole with $r_{+}=r_{c}$, respectively. Note that the orange, green, and purple lines meet at the point $P$, which marks the minimum value of $b$ and the maximum value of $Q$ in the yellow region. The solid blue line corresponds to a near-extremal black hole with the charge $Q=0.900 Q_{\text {ext }}$, where $Q_{\text {ext }}$ is the charge of the extremal black hole.

with $\Delta(Q)=\frac{8 \pi}{3} \sqrt{\frac{b}{\pi}} Q^{3 / 2} \Gamma\left(\frac{1}{4}\right)^{2}$, which leads to $M<$ $\Delta(Q)$.

(iii) $f\left(r_{\text {min }}\right)<0$ : The local minimum value of $r f(r)$ at $r=r_{\min }$ must be negative. When $f\left(r_{\min }\right)=0$, we have the extremal black hole solution with $r_{-}=r_{+}$. For later use, $Q_{\text {ext }}$ denotes the charge of the extremal black hole.

(iv) $f\left(r_{\max }\right)>0$ : The local maximum value of $r f(r)$ at $r=r_{\max }$ must be positive. When $f\left(r_{\max }\right)=0$, we have the Nariai black hole solution with $r_{+}=r_{c}$, which could only be calculated numerically [64].

The above conditions together give the allowed region in the parameter space, in which a BI-dS black hole has three horizons. We also find that there exists a lower bound $b_{\text {min }}$ on $b$ and an upper bound $Q_{\max }$ on $Q$ in the allowed region. In fact, it can show that the boundaries $b Q=\frac{1}{2}, M=\Delta(Q)$, and $f\left(r_{\min }\right)=0$ can intersect at one point in the $b-Q$ plane, which gives

$$
b_{\min }=\frac{2 \sqrt{2 \pi} \Gamma\left(\frac{1}{4}\right)^{2}}{3 M}, \quad Q_{\max }=\frac{3 M}{4 \sqrt{2 \pi} \Gamma\left(\frac{1}{4}\right)^{2}} .
$$

We plot the allowed region and their boundaries in the $b-Q$ parameter space in Fig. 1, where $M=16 \pi$ and $\Lambda=0.14$.

\section{QUASINORMAL MODE}

In this section, we discuss the QNMs for a charged and massive scalar field in a BI-dS black hole. We first consider 
a scalar perturbation of mass $\mu$ and charge $q$ governed by the Klein-Gordon equation

$$
\left(\mathbf{D}^{2}-\mu^{2}\right) \Phi=0,
$$

where $\mathbf{D}$ denotes the covariant derivative $\mathbf{D}=\nabla-i q \mathbf{A}$. To facilitate our numerical calculation, we will use Eddington-Finkelstein ingoing coordinates $(v, r, \theta, \phi)$ with $v=t+r_{*}$, where $r_{*}$ is the tortoise coordinate defined as $d r_{*}=d r / f(r)$. In addition, we choose an appropriate gauge transformation such that $\mathbf{A}=A_{v} d v=-\frac{Q}{r}{ }_{2} \mathcal{F}_{1}$ $\left[\frac{1}{4}, \frac{1}{2}, \frac{5}{4},-\frac{Q^{2}}{\beta^{2} r^{4}}\right] d v$. Since the BI-dS black hole solution is static and spherically symmetry, a mode solution of Eq. (3.1) can have the separable form

$$
\Phi=e^{-i \omega v} Y_{l m}(\theta, \phi) \psi_{\omega l}(r),
$$

where $Y_{l m}(\theta, \phi)$ is the harmonic function of the unit two sphere. Plugging Eq. (3.2) into Eq. (3.1), we obtain the radial equation

$$
\begin{aligned}
0= & {\left[r^{2} f \partial_{r}^{2}+\left(r^{2} f^{\prime}+2 r f-2 i q A_{v} r^{2}-2 i \omega r^{2}\right) \partial_{r}\right.} \\
& \left.-2 i \omega r-2 i q r A_{v}-i q r^{2} \partial_{r} A_{v}-l(l+1)-\mu^{2} r^{2}\right] \psi_{\omega l}(r),
\end{aligned}
$$

where $f^{\prime}$ denotes $d f(r) / d r$. One can perform the Frobenius method to obtain the solutions near the event and cosmological horizons, respectively. In fact, we define a new coordinate $x \equiv\left(r-r_{+}\right) /\left(r_{c}-r_{+}\right)$. Near the event horizon, i.e., $x \rightarrow 0, \psi_{\omega l}(r)$ has the ingoing and outgoing boundary solutions,

$$
\psi_{\omega l}^{\text {ingoing }} \sim \text { const, } \quad \psi_{\omega l}^{\text {outgoing }} \sim x^{i \frac{\omega+\left.q A_{v}\right|_{r=r_{+}}}{\kappa_{+}}} .
$$

And near the cosmological horizon, i.e., $x \rightarrow 1, \psi_{\omega l}(r)$ also has the ingoing and outgoing boundary solutions,

$\psi_{\omega l}^{\text {ingoing }} \sim$ const, $\quad \psi_{\omega l}^{\text {outgoing }} \sim(1-x)^{-i \frac{\omega+q A_{v} \mid r=r_{c}}{\kappa_{c}}}$.

Here $\kappa_{h} \equiv\left|f^{\prime}\left(r_{h}\right)\right| / 2$ with $h \in\{+,-, c\}$ is the surface gravity at each horizon.

Imposing the ingoing boundary condition at the event horizon and the outgoing boundary condition at the cosmological horizon on Eq. (3.3) selects a set of discrete frequencies $\omega_{\ln }(n=1,2, \ldots)$, called QNMs [22]. There are many analytic and numerical ways to extract QNMs [22,23]. Here we employ the Chebyshev collocation scheme and the associated Mathematica package developed in [65-67]. We redefine field $\psi_{\omega l}$ adapted to our numerical scheme

$$
\psi_{\omega l}=\frac{1}{x}(1-x)^{-i \frac{\omega+q A_{v} \mid}{\kappa_{c}=r_{c}}} \phi_{\omega l},
$$

where the new field $\phi_{\omega l}$ becomes regular at both the event and cosmological horizons. After the radial equation for $\phi_{\omega l}$ is obtained, we can use the Mathematica package to find a series of QNMs, $\omega_{\mathrm{ln}}$. The spectral gap $\alpha$ in Eq. (1.1) is then given by $\alpha=\inf _{\ln }\left\{-\operatorname{Im}\left(\omega_{\ln }\right)\right\}$.

\section{NUMERICAL RESULTS}

In this section, we present the numerical results about the low-lying QNMs for a scalar field and check the validity of SCC. The results shown in this section are obtained with the Mathematica package of [65-67] and checked with some QNMs given in [68], where the WKB approximation was used. Since SCC may be violated near extremality in a $\mathrm{RN}$-dS black hole, we here focus on the near-extremal parameter space of a BI-dS black hole.

In the Mathematica package of [65-67], the spectrum of QNMs is computed by discretizing the QNMs equations with the pseudospectral method and solving the resulting generalized eigenvalue equation. The key idea is to turn the problem of solving a linear ordinary differential equation into finding eigenvalues and eigenfunctions of some matrix. This method only assumes that the eigenfunctions are analytical and does not require the ansatz of the eigenfunctions or an initial guess. On the other hand, although the WKB approximation method is powerful to give accurate results for the complex modes, it is known that the infinite set of purely imaginary modes are missed by this method since the ansatz of the WKB approximation misses the purely imaginary modes [22]. In some cases, this type of modes may become the most physically relevant modes (e.g., nearextremal $[\mathrm{NE}]$ modes in the near-extremal limit as discussed below). Moreover, the WKB approximation assumes that the potential has a single extremum, which may fail in some cases [69].

In Table I, we show the lowest-lying QNMs $\omega / \kappa_{-}$of some representative points in the relevant parameter region. Note that some results in $[30,31]$ are recovered in the large $b$ limit as expected. Besides, when the scalar field is charged, the symmetry between left and right modes is broken due to the presence of scalar charge, which was also observed in [31,32]. Similar to the RN-dS black hole case, we find that the violation of SCC occurs when the black hole lies close enough to extremality (e.g., $Q / Q_{\text {ext }}=0.996$ ). Interestingly, it shows that a smaller value of $b$ tends to decrease the absolute value of $\operatorname{Im}(\omega) / \kappa_{-}$, which can alleviate the violation of SCC and even save SCC. Note that we set $M=16 \pi$ without loss of generality in this section.

\section{A. Neutral scalar field}

Recently, the authors of [30] found three qualitatively different families of QNMs for a RN-dS black hole: the photon sphere (PS) family, which can be traced back to the photon sphere, the de Sitter (dS)family, which is deformation of the pure de Sitter modes, and the near-extremal (NE) 
TABLE I. The lowest-lying QNMs $\omega / \kappa_{-}$for a massless scalar field of charge $q$ in a near-extremal BI-dS black hole. In the large $b$ limit, i.e., $b=10000$, the values of $\omega / \kappa_{-}$with $\Lambda=0.02, Q / Q_{\text {ext }}=0.991, q=0.1$ and $\Lambda=0.06, Q / Q$ ext $=0.996, q=0$ are consistent with those in a RN-dS black hole obtained in [30,31], respectively.

\begin{tabular}{|c|c|c|c|c|c|c|}
\hline$\Lambda$ & $b$ & $Q / Q_{\mathrm{ext}}$ & $q$ & $l=0$ & $l=1$ & $l=10$ \\
\hline \multirow{8}{*}{0.02} & \multirow{5}{*}{0.5} & \multirow{2}{*}{0.991} & 0 & 0 & $\pm 0.586851-0.102835 i$ & $\pm 4.107680-0.099091 i$ \\
\hline & & & 0.1 & $0.015305+0.001095 i$ & $0.686126-0.101651 i$ & $4.204171-0.098960 i$ \\
\hline & & \multirow{2}{*}{0.996} & 0 & 0 & $\pm 2.514067-0.411424 i$ & $\pm 17.649019-0.393928 i$ \\
\hline & & & 0.1 & $0.065124+0.004991 i$ & $2.948696-0.400322 i$ & $18.072264-0.392465 i$ \\
\hline & & \multirow{2}{*}{0.991} & 0 & 0 & $-0.475688 i$ & $\pm 14.365381-0.491756 i$ \\
\hline & \multirow{3}{*}{10000} & & 0.1 & $0.057773+0.002229 i$ & $0.032203-0.475118 i$ & $-14.080016-0.491441 i$ \\
\hline & & \multirow{2}{*}{0.996} & 0 & 0 & $-0.789379 i$ & $\pm 23.969407-0.808962 i$ \\
\hline & & & 0.1 & $0.096356+0.003870 i$ & $0.053708-0.788423 i$ & $-23.488922-0.808825 i$ \\
\hline \multirow{8}{*}{0.06} & \multirow{4}{*}{0.5} & \multirow{2}{*}{0.991} & 0 & 0 & $\pm 0.686276-0.121754 i$ & $\pm 4.863532-0.116340 i$ \\
\hline & & & 0.1 & $0.039490+0.002376 i$ & $0.817179-0.119918 i$ & $4.990056-0.116174 i$ \\
\hline & & \multirow{2}{*}{0.996} & 0 & 0 & $\pm 2.520908-0.414697 i$ & $\pm 17.902524-0.394830 i$ \\
\hline & & & 0.1 & $0.143437+0.009706 i$ & $3.010856-0.402037 i$ & $18.376723-0.393336 i$ \\
\hline & \multirow{4}{*}{10000} & \multirow{2}{*}{0.991} & 0 & 0 & $\pm 1.930716-0.481345 i$ & $\pm 13.798347-0.462716 i$ \\
\hline & & & 0.1 & $0.127461+0.001895 i$ & $2.265562-0.474726 i$ & $14.119498-0.462581 i$ \\
\hline & & \multirow{2}{*}{0.996} & 0 & 0 & $\pm 3.242616-0.795833 i$ & $\pm 23.189760-0.764924 i$ \\
\hline & & & 0.1 & $0.213619+0.003591 i$ & $3.808829-0.781460 i$ & $23.733891-0.764259 i$ \\
\hline
\end{tabular}

family, which only appears for near-extremal black holes. Similarly, we also observe these three distinct families for a neutral massless scalar field in a near-extremal BI-dS black hole. In Fig. 2, we plot the dominant modes of each of the families divided by $\kappa_{-}$. Specifically, $\operatorname{Im}(\omega) / \kappa_{-}$is plotted against $Q / Q_{\text {ext }}$ for various values of $b$ and $\Lambda$ in Fig. 2(a). As shown in Fig. 1, for a fixed value of $b$ not far from $b_{\min }$, the $M=\Delta(Q)$ line puts a lower bound on $Q / Q_{\text {ext }}$, which is depicted as the solid vertical lines in Fig. 2(a). It is noteworthy that all $\operatorname{Im}(\omega) / \kappa_{-}$go to zero as $Q / Q_{\text {ext }}$ approaches its lower bound. Indeed, in the limit of $M \rightarrow \Delta(Q)$, the Cauchy horizon radius $r_{-}$goes to zero, and hence the surface gravity at the Cauchy horizon $\kappa_{-}$becomes

$$
\kappa_{-}=\left|\frac{1}{2 r} \frac{d(r f(r))}{d r}\right|_{r=r_{-}} \mid \cong \frac{5 b Q}{3 r_{-}} \rightarrow \infty,
$$

where we use Eq. (2.5). Since QNMs are still finite in this limit, we find that $\operatorname{Im}(\omega) / \kappa_{-}=0$ when $M=\Delta(Q)$ (i.e., solid vertical lines in Fig. 2 and dashed green lines in Figs. 1 and 3). Moreover, Fig. 2(a) shows that, when $Q / Q_{\text {ext }}$ increases toward the extremal limit, the $\operatorname{Im}(\omega) / \kappa_{-}$for the three families' dominant modes all decreases. In the extremal limit, the PS and dS families become divergent while the NE family approaches -1 from below and hence takes over to make $1 / 2<\beta<1$. Thus, with fixed values of $b$ and $\Lambda$, the presence of NE mode can invalidate SCC as long as the black hole lies close enough to extremality. Moreover, the dS family is more sensitive to $\Lambda$ than the PS and NE families and can become dominant for "small" black holes (small $\Lambda$ ). Moreover, it shows that the range of $Q / Q_{\text {ext }}$, where SCC is violated, shrinks with decreasing value of $b[b$ decreases from the left column to the right column in Fig. 2(a)]. To better illustrate the dependence of $\operatorname{Im}(\omega) / \kappa_{-}$on $b$, we plot
$\operatorname{Im}(\omega) / \kappa_{-}$against $b$ for various values of $Q / Q_{\text {ext }}$ and $\Lambda$ in Fig. 2(b). It is expected that SCC is easier to be violated when the black hole is closer to extremality. In fact, increasing $Q / Q_{\text {ext }}$ toward extremality from the left column to the right column in Fig. 2(b), we find that the SCC violation ranges of $b$, which are on the left of the dashed vertical lines, increase in size. Note that there is no SCC violation in the $Q / Q_{\text {ext }}=$ 0.991 case. In Fig. 1, it shows that the $Q / Q_{\text {ext }}$-constant line (e.g., the blue line with $Q / Q_{\text {ext }}=0.900$ ) always has a lower bound $b_{Q / Q_{\text {ext }}}$ on $b$, which is also imposed by the $M=\Delta(Q)$ line. The solid vertical lines in Fig. 2(b) represent $b=b_{Q / Q_{\text {ext }}}$, on which $M=\Delta(Q)$ and thus $\beta=0$. So with fixed values of $Q / Q_{\text {ext }}$ and $\Lambda$, one can always have $\beta<1 / 2$ when $b$ is close enough to $b_{Q / Q_{\text {ext }}}$. In the $Q / Q_{\text {ext }}=0.995$ and $Q / Q_{\text {ext }}=0.999$ cases, $\mathrm{SCC}$ is violated for large enough values of $b$. Nevertheless, we can recover SCC by making $b$ close enough to $b_{Q / Q_{\text {ext }}}$.

Finally, we depict the density plot of $\beta$ in the small $b$ region in Fig. 3, where the solid black line represents the threshold $\beta=1 / 2$. So, SCC is violated in the region between the extremal line (dashed orange) and $\beta=1 / 2$ (solid black). The $Q / Q_{\text {ext }}=0.995$ line is displayed as a red line, which also shows that SCC can be recovered for a small enough value of $b$. For a near-extremal BI-dS black hole with a constant charge $Q$, it also shows in Fig. 3 that $\mathrm{SCC}$ is respected when $b$ is close enough to the dashed green line. Furthermore, Fig. 3 displays that SCC can be recovered for a highly extremal BI-dS black hole as long as the Born-Infeld parameter $b$ is sufficiently close to $b_{\min }$ (the point $P$ ).

\section{B. Charged scalar field}

We now turn on the charge of a scalar field and investigate the validity of SCC. In Fig. 4, we first plot 

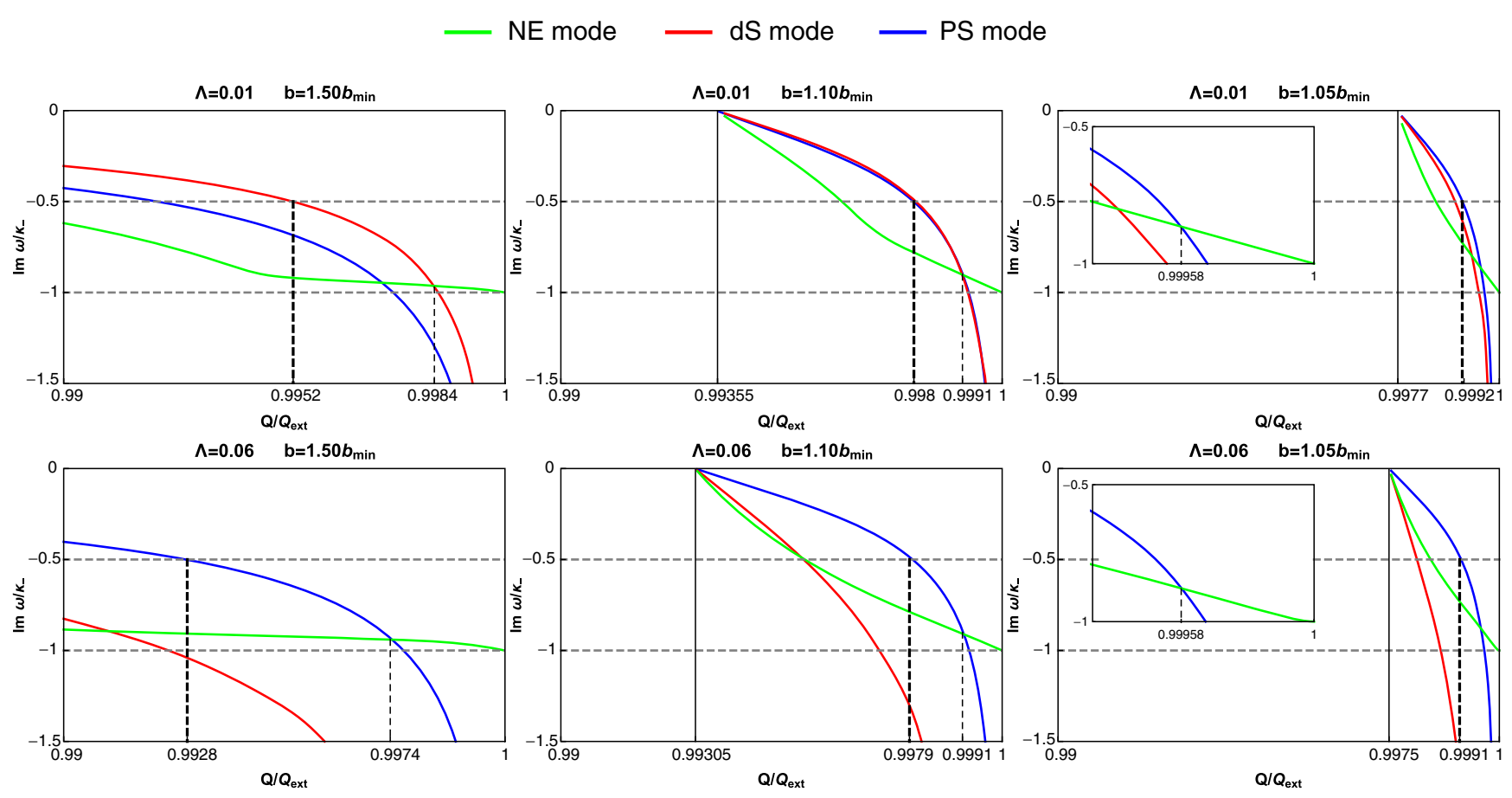

(a)
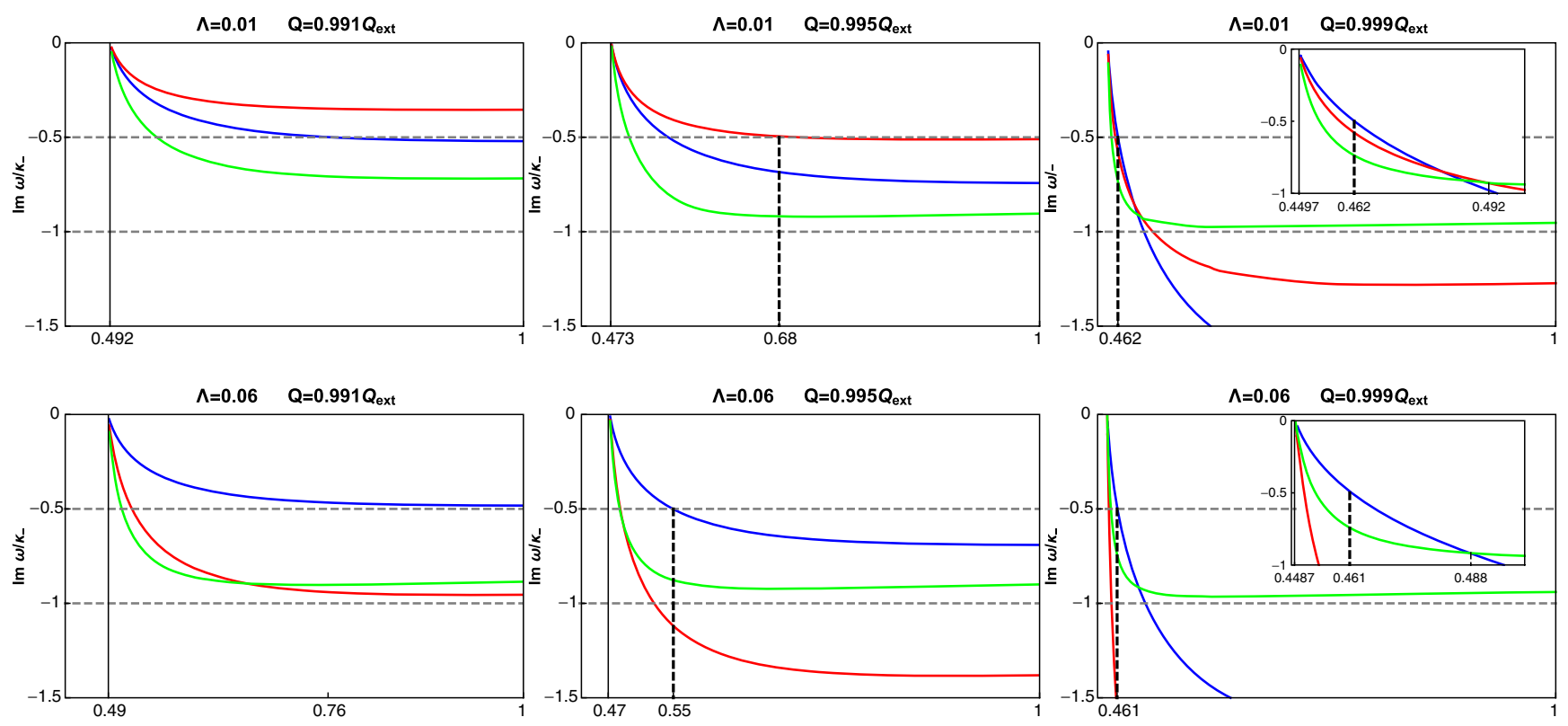

(b)

FIG. 2. Dominant modes of three families for a neutral massless scalar field, showing the dominant NE mode (green) at $l=0$, the dominant dS mode (red) at $l=1$, and the (nearly) dominant complex PS mode (blue) at $l=10$. The thick and thin dashed black vertical lines designate the points where $\beta \equiv-\operatorname{Im}(\omega) / \kappa_{-}=1 / 2$ and where the $\mathrm{NE}$ modes become dominant, respectively. (a) Dominant modes of three families for various values of $b$ and $\Lambda$ with varying $Q / Q_{\text {ext }}$. The solid vertical lines indicate the minimal charge ratio, below which a BI-dS black hole can not possess the Cauchy horizon. Note that the minimal charge ratio lines in the left column lie out of the relevant region. It shows that the SCC violation region, in which $\max \left\{\operatorname{Im}(\omega) / \kappa_{-}\right\}<-1 / 2, \operatorname{decreases}$ in size as $b$ decreases. (b) Dominant modes of three families for various values of $Q / Q_{\text {ext }}$ and $\Lambda$ with varying $b$. The solid vertical lines indicate the minimal $b$, below which a BI-dS black hole can not possess the Cauchy horizon. It shows that SCC can be saved for a small enough value of $b$. 


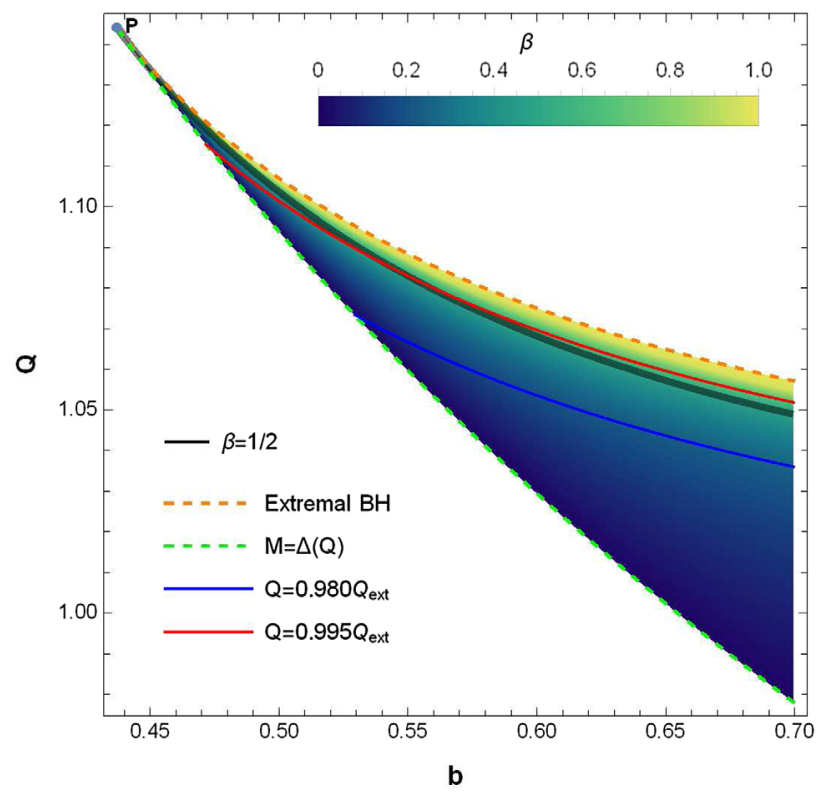

FIG. 3. Density plot of $\beta$ for a neutral massless scalar field in a BI-dS black hole with $\Lambda=0.06$. The parameter space of interest is bounded by $M=\Delta(Q)$ (dashed green) and the extremal black hole (dashed orange). Note that $\beta=1 / 2$ on the thick black line. So, SCC is violated in the region above the thick black line. Two lines in red and blue denote the near-extremal black holes with the charge $Q / Q_{\text {ext }}=0.995$ and $Q / Q_{\text {ext }}=0.980$, respectively. In the $Q / Q_{\mathrm{ext}}=0.995$ case, $\mathrm{SCC}$ is violated in some parameter region but can be saved when $b$ is small enough.

the lowest-lying QNMs as a function of the scalar charge $q$ for a massless charged scalar field in a BI-dS black hole, which behaves rather similarly to the $\mathrm{RN}-\mathrm{dS}$ black hole case. The blue lines represent the $l=0$ zero mode, which reduces to a trivial mode in the limit $q \rightarrow 0$. In particular, we observe the presence of superradiant instability in the small scalar charge regime. This linear instability suggests that the perturbations will be severely unstable even in the exterior of the black hole, and thus one cannot infer anything about SCC when superradiance occurs. Note that the nonsmoothness of the blue lines around $q \sim 1.5$ is caused by the competition between the PS and NE modes. The $b=1$ case is shown in the left panel of Fig. 4, which shows that SCC is violated for $q=0$ since the $l=0$ zero trivial mode is discarded. However, for a nonzero $q$, SCC is saved out due to the nontrivial $l=0$ zero mode. The right panel of Fig. 4 displays that, for a smaller value of $b=0.5$, the higher $l$-modes are also above the threshold line $\beta=1 / 2$, and the superradiant regime increases in size, which means that small $b$ tends to make the black hole more unstable. From Fig. 4 , we see that $\beta$ is determined by the $l=0$ dominant mode for a charged scalar field.

Further increasing the black hole charge $Q$ toward extremality, we plot the $l=0$ dominant mode as a function of the scalar charge $q$ for a massless charged scalar field in a BI-dS black hole in Fig. 5. The dependence of the $l=0$ dominant mode on $b$ is plotted in the left panel of Fig. 5, where $Q / Q_{\text {ext }}=1-10^{-4}$. The curve with $b=10$ is almost identical to the RN-dS case, which was shown in Fig. 11 of [31]. It shows that the SCC violation occurs for $b=10,1$, and 0.5 in some scalar charge regime. Nevertheless, these violation regions decrease in size as $b$ decreases. Interestingly, SCC is always respected when $b=0.45$. In the right panel of Fig. 5, we plot the $l=0$ dominant mode for a more extremal BI-dS black hole with $Q / Q_{\text {ext }}=1-10^{-6}$ and display that the "wiggles," i.e., small oscillations around $\beta=1 / 2$, appear in the $b=10,1$, and 0.5 cases. It is noteworthy that the wiggles disappear, and SCC is restored when $b \lesssim 0.46$.

Next, we turn to the dependence of the $l=0$ dominant mode on $\Lambda$ in Fig. 6, where the $l=0$ dominant mode is plotted as a function of $\Lambda$ for various values of $q$ and $b$. Note that $\Lambda$ is bounded above by a maximum value due to the Nariai limit of a BI-dS black hole. In the Nariai limit,
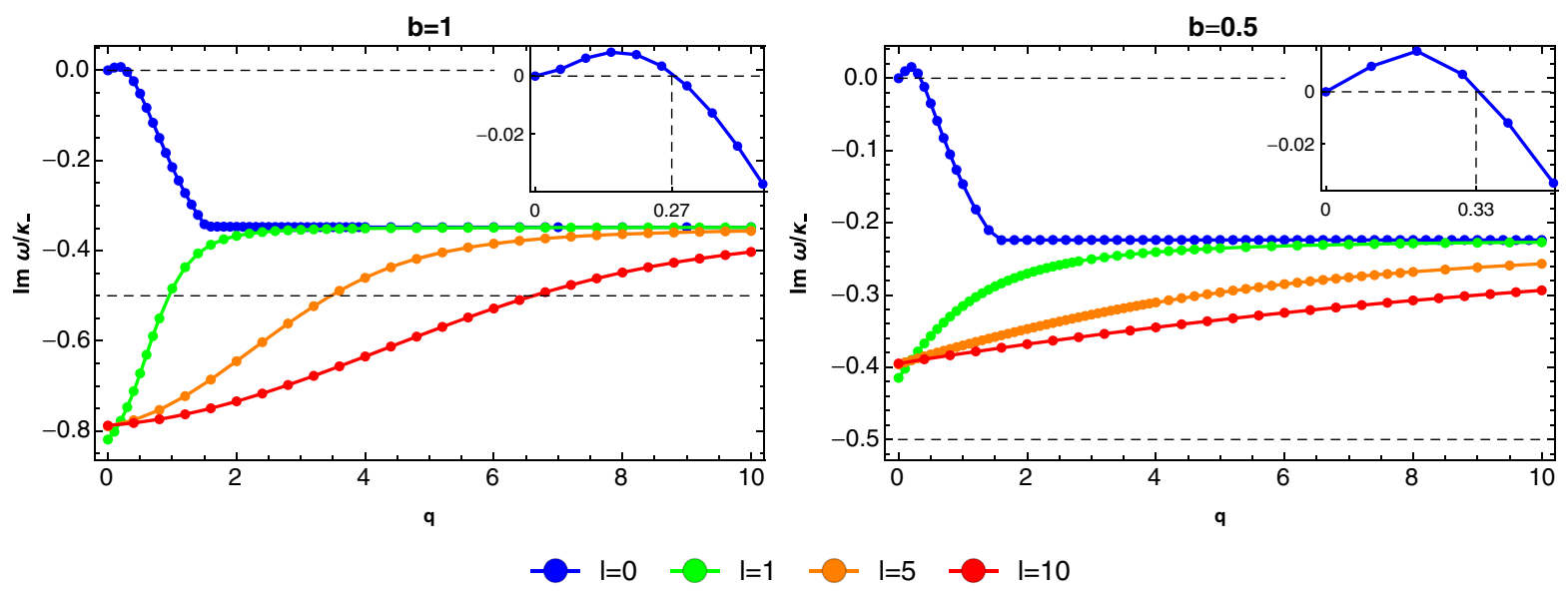

FIG. 4. Plots of the lowest-lying QNMs as a function of the scalar charge $q$ for a massless charged scalar field in a BI-dS black hole with $\Lambda=0.06$ and $Q / Q_{\text {ext }}=0.996$. Left: $b=1$. Right: $b=0.5$. The dashed vertical line designates an upper bound on the scalar charge, under which superradiance is present. 

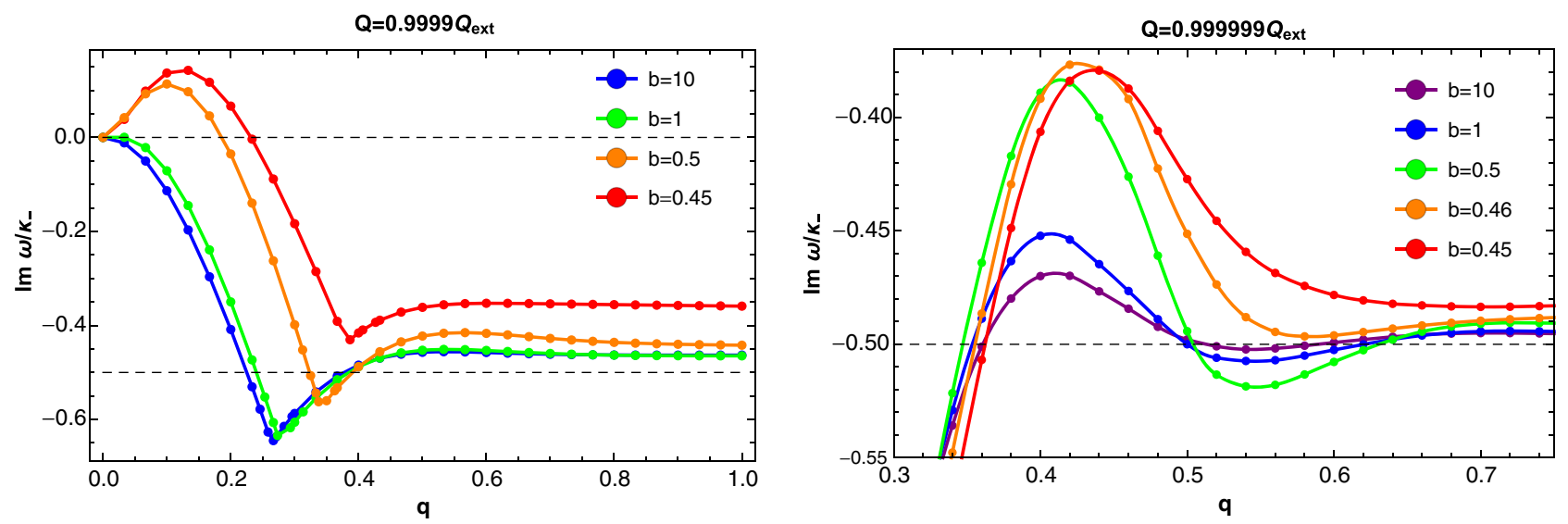

FIG. 5. Plots of the $l=0$ dominant mode as a function of the scalar charge $q$ for a massless charged scalar field in a BI-dS black hole with $\Lambda=0.14$. Left: $Q / Q_{\text {ext }}=1-10^{-4}$. The SCC violation region decreases in size and even disappears with decreasing $b$. Right: $Q / Q_{\text {ext }}=1-10^{-6}$. The presence of the wiggles for large values of $b(b=10,1$ and 0.5$)$ and absence of the wiggles for small values of $b(b=0.46$ and 0.45$)$ are observed.
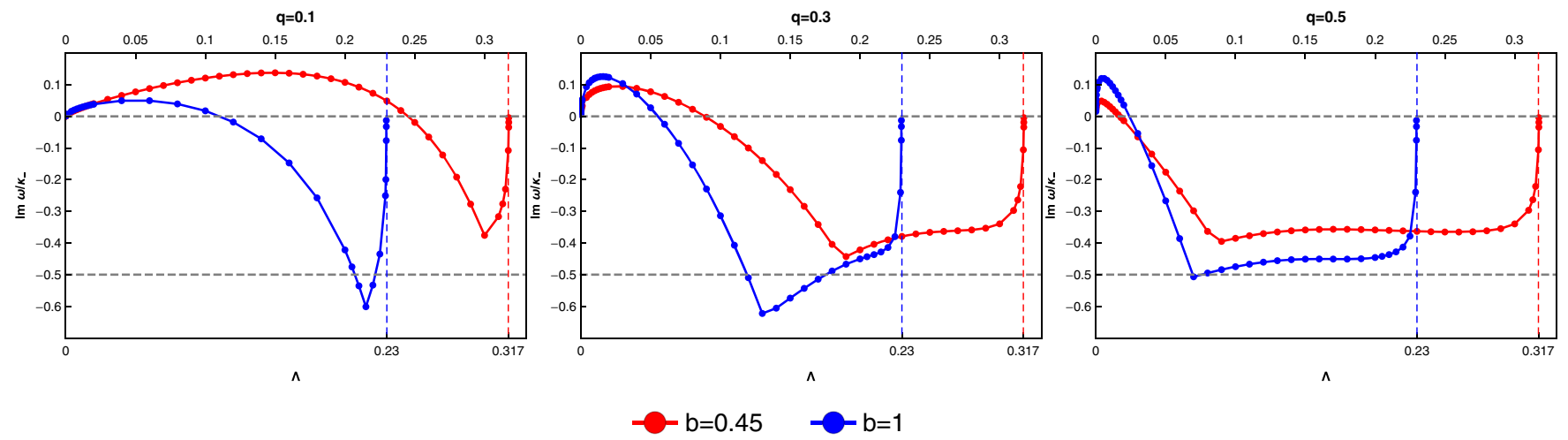

FIG. 6. Plots of the $l=0$ dominant mode for a massless charged scalar field as a function of the cosmological constant $\Lambda$ in a BI-dS black hole with $Q / Q_{\text {ext }}=1-10^{-4}$ for the scalar charge $q=0.1,0.3$, and 0.5 . The dashed vertical blue and red lines represent the maximum values of $\Lambda$ for $b=1$ and $b=0.45$, respectively. SCC is respected for the smaller $b$ (i.e., $b=0.45$ ) while there exists the $\mathrm{SCC}$ violation region for the larger $b$ (i.e., $b=1$ ).
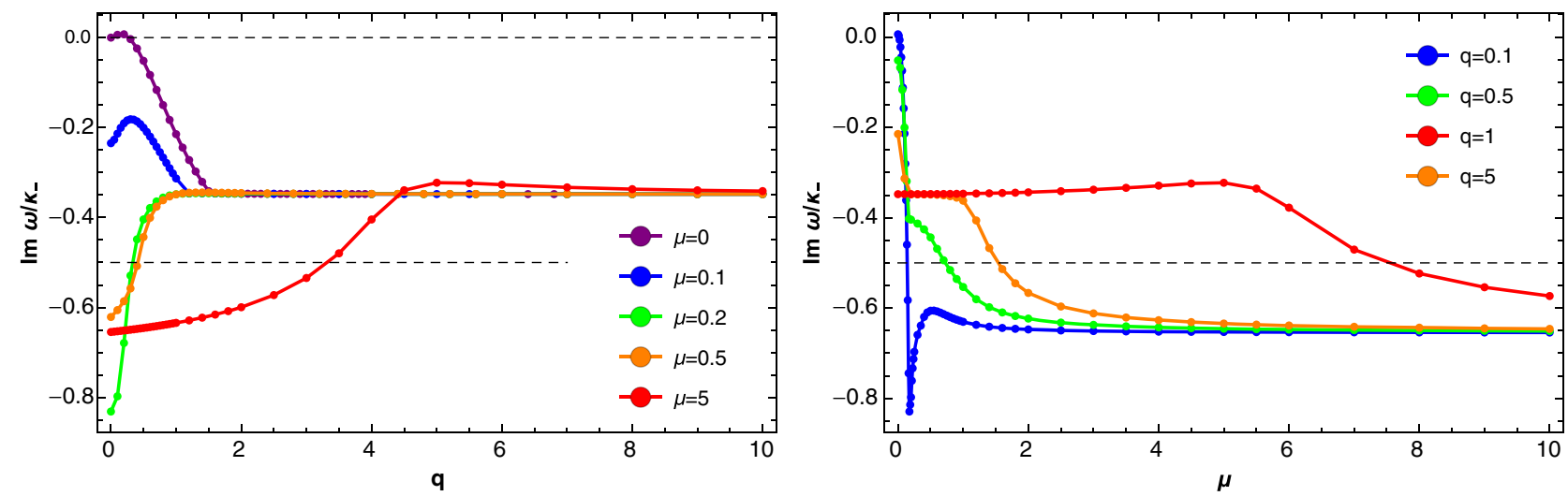

FIG. 7. Plots of the $l=0$ dominant mode for a massive charged scalar field of charge $q$ and mass $\mu$ in a BI-dS black hole with $\Lambda=0.06, Q / Q_{\mathrm{ext}}=0.996$, and $b=1$. Left: as a function of $q$ for various values of $\mu$. Right: as a function of $\mu$ for various values of $q$. It shows that SCC is violated more easily for a more massive scalar. 
$\operatorname{Im}(\omega)$ approaches zero while $\kappa_{-}$stays finite, which explains $\operatorname{Im}(\omega) / \kappa_{-}=0$ at the maximum value of $\Lambda$ shown in Fig. 6. Again, the nonsmoothness in Fig. 6 results from the competition between the PS and NE modes. When $b=1, \mathrm{SCC}$ can be violated in some parameter region of $\Lambda$ and $q$. For a smaller $b=0.45$, these violation regions all disappear.

Finally, we investigate the dependence of the $l=0$ dominant mode on the scalar mass $\mu$ in Fig. 7. As shown in the left panel in Fig. 7, the superradiant instability is highly sensitive to the scalar mass. For a sufficiently large value of $\mu$, superradiant instability no longer exists. Moreover, the $\operatorname{Im}(\omega) / \kappa_{-}$of the dominant $l=0$ mode can be smaller than $-1 / 2$ for large enough $\mu$. It also displays, in the right panel of Fig. 7, that the $l=0$ dominant mode for various values of $q$ all go below the threshold line when the scalar field is sufficiently massive. So, Fig. 7 shows that SCC tends to be violated for a larger scalar mass. On the other hand, it also shows that SCC tends to be saved for a larger scalar charge.

\section{CONCLUSION}

In this paper, we investigated the validity of SCC in a BI-dS black hole perturbed by a scalar field with/without a charge. After the parameter region, where a BI-dS black hole can possess the Cauchy horizon, was obtained in Sec. II, we presented the numerical results for a neutral scalar field in Sec. IV A and a charged scalar in Sec. IV B.

For the Born-Infeld parameter $b \gtrsim 1$, the behavior of SCC in a BI-dS black hole is quite similar to that in a RNdS black hole. In fact, we observed that SCC is always violated when a BI-dS black hole is sufficiently close to extremality in the neutral case, and the SCC violation region, especially the wiggles, can appear in the charged case. On the other hand, for a smaller value of $b$, the NLED effect can play an important role and tends to alleviate the violation of SCC. Specifically, we found that (i) For a massless neutral scalar field, Fig. 2 showed that the SCC violation region decreases in size with deceasing $b$.

(ii) For a massless neutral scalar field, Fig. 3 showed that SCC can always be restored for a near-extremal BI-dS black hole with a fixed charge ratio $Q / Q_{\text {ext }}$ or a charge $Q$ when $b$ is sufficiently small.

(iii) For a massless charged scalar, Figs. 5 and 6 showed that the SCC violation region also decreases in size with deceasing $b$. Moreover, the violation region can disappear for a sufficiently small value of $b$.

The dependence of SCC on the scalar mass and charge was discussed in Fig. 7 for a massive charged scalar, which showed that the smaller the scalar mass is (or the larger the scalar charge is), the easier it becomes to restore SCC. Our results indicate that the quantum effects could play a crucial role in rescuing SCC. Therefore, it is inspiring to check the validity of SCC in modified gravity theories, even a quantum gravity model.

Finally, we discuss the limitation of our analysis. First, we only considered the scalar field perturbation on the fixed BI-dS black hole background in the probe limit without taking into account the backreaction of the scalar field on the black hole spacetime. So, our results on stability actually refer to the scalar field rather than the BI-dS black hole spacetime. Second, the probe limit is trustworthy as long as the charge and mass of the scalar field are small enough. Therefore, the results for the scalar field with a large charge or mass (e.g., lessen the SCC violation when the charge of the scalar field is large) may not be reliable in the probe limit.

\section{ACKNOWLEDGMENTS}

We are deeply grateful to Hongbao Zhang for his excellent presentations and valuable comments. We also thank Haitang Yang for his helpful discussions and suggestions. This work is supported in part by NSFC (Grants No. 11005016, No. 11875196, and No. 11375121).
[1] R. Penrose, Gravitational collapse: The role of general relativity, Riv. Nuovo Cimento 1, 252 (1969) [Gen. Relativ. Gravit. 34, 1141 (2002)].

[2] S. W. Hawking and R. Penrose, The singularities of gravitational collapse and cosmology, Proc. R. Soc. A 314, 529 (1970).

[3] R. Penrose, Gravitational Collapse and Space-Time Singularities, Phys. Rev. Lett. 14, 57 (1965).

[4] J. Luk and S.-J. Oh, Strong cosmic censorship in spherical symmetry for two-ended asymptotically flat initial data I. The interior of the black hole region, arXiv:1702.05715.
[5] J. Luk and S.-J. Oh, Strong cosmic censorship in spherical symmetry for two-ended asymptotically flat initial data II. The exterior of the black hole region, arXiv:1702.05716.

[6] D. Christodoulou, The formation of black holes in general relativity, in On Recent Developments in Theoretical and Experimental General Relativity, Astrophysics and Relativistic Field Theories. Proceedings, 12th Marcel Grossmann Meeting on General Relativity, Paris, France, 2009 (2008), Vol. 1-3, pp. 24-34, http://inspirehep.net/record/786592.

[7] C. M. Chambers, The Cauchy horizon in black hole de Sitter space-times, Ann. Isr. Phys. Soc. 13, 33 (1997). 
[8] M. Dafermos, The interior of charged black holes and the problem of uniqueness in general relativity, Commun. Pure Appl. Math. 58, 0445 (2005).

[9] E. Poisson and W. Israel, Internal structure of black holes, Phys. Rev. D 41, 1796 (1990).

[10] A. Ori, Inner Structure of a Charged Black Hole: An Exact Mass-Inflation Solution, Phys. Rev. Lett. 67, 789 (1991).

[11] S. Hod and T. Piran, Mass Inflation in Dynamical Gravitational Collapse of a Charged Scalar Field, Phys. Rev. Lett. 81, 1554 (1998).

[12] P. R. Brady and J. D. Smith, Black Hole Singularities: A Numerical Approach, Phys. Rev. Lett. 75, 1256 (1995).

[13] R. H. Price, Nonspherical perturbations of relativistic gravitational collapse. 1. Scalar and gravitational perturbations, Phys. Rev. D 5, 2419 (1972).

[14] M. Dafermos, I. Rodnianski, and Y. Shlapentokh-Rothman, Decay for solutions of the wave equation on Kerr exterior spacetimes III: The full subextremal case $|a|<M$, arXiv: 1402.7034 .

[15] Y. Angelopoulos, S. Aretakis, and D. Gajic, Late-time asymptotics for the wave equation on spherically symmetric, stationary spacetimes, Adv. Math. 323, 529 (2018).

[16] M. Dafermos, Black holes without spacelike singularities, Commun. Math. Phys. 332, 729 (2014).

[17] J.-F. Bony and D. Hafner, Decay and non-decay of the local energy for the wave equation in the De Sitter-Schwarzschild metric, arXiv:0706.0350.

[18] S. Dyatlov, Asymptotics of linear waves and resonances with applications to black holes, Commun. Math. Phys. 335, 1445 (2015).

[19] S. Dyatlov, Asymptotic distribution of quasi-normal modes for Kerr-de Sitter black holes, Ann. Inst. Henri Poincaré 13, 1101 (2012).

[20] P. Hintz and A. Vasy, The global non-linear stability of the Kerr-de Sitter family of black holes, arXiv:1606.04014.

[21] P. Hintz, Non-linear stability of the Kerr-Newman-de Sitter family of charged black holes, arXiv:1612.04489.

[22] E. Berti, V. Cardoso, and A. O. Starinets, Quasinormal modes of black holes and black branes, Classical Quantum Gravity 26, 163001 (2009).

[23] R. A. Konoplya and A. Zhidenko, Quasinormal modes of black holes: From astrophysics to string theory, Rev. Mod. Phys. 83, 793 (2011).

[24] P. R. Brady, C. M. Chambers, W. Krivan, and P. Laguna, Telling tails in the presence of a cosmological constant, Phys. Rev. D 55, 7538 (1997).

[25] J. L. Costa, P. M. Girão, J. Natário, and J. Drumond Silva, On the global uniqueness for the Einstein-Maxwell-scalar field system with a cosmological constant. Part 3: Mass inflation and extendibility of the solutions, Commun. Math. Phys. 339, 903 (2015).

[26] J. L. Costa, P. M. Girão, J. Natário, and J. Drumond Silva, On the global uniqueness for the Einstein-Maxwell-scalar field system with a cosmological constant: I. Well posedness and breakdown criterion, Classical Quantum Gravity 32, 015017 (2015).

[27] J. L. Costa, P. M. Girão, J. Natário, and J. Drumond Silva, On the global uniqueness for the Einstein-Maxwell-scalar field system with a cosmological constant, Commun. Math. Phys. 339, 903 (2015).
[28] P. Hintz and A. Vasy, Analysis of linear waves near the Cauchy horizon of cosmological black holes, J. Math. Phys. (N.Y.) 58, 081509 (2017).

[29] C. Kehle and Y. Shlapentokh-Rothman, A scattering theory for linear waves on the interior of ReissnerNordström black holes, Ann. Inst. Henri Poincaré 20, 1583 (2019).

[30] V. Cardoso, J. L. Costa, K. Destounis, P. Hintz, and A. Jansen, Quasinormal Modes and Strong Cosmic Censorship, Phys. Rev. Lett. 120, 031103 (2018).

[31] Y. Mo, Y. Tian, B. Wang, H. Zhang, and Z. Zhong, Strong cosmic censorship for the massless charged scalar field in the Reissner-Nordstrom-de Sitter spacetime, Phys. Rev. D 98, 124025 (2018).

[32] O. J. C. Dias, H. S. Reall, and J. E. Santos, Strong cosmic censorship for charged de Sitter black holes with a charged scalar field, Classical Quantum Gravity 36, 045005 (2019).

[33] V. Cardoso, J. L. Costa, K. Destounis, P. Hintz, and A. Jansen, Strong cosmic censorship in charged black-hole spacetimes: Still subtle, Phys. Rev. D 98, 104007 (2018).

[34] S. Hod, Strong cosmic censorship in charged black-hole spacetimes: As strong as ever, Nucl. Phys. B941, 636 (2019).

[35] B. Ge, J. Jiang, B. Wang, H. Zhang, and Z. Zhong, Strong cosmic censorship for the massless Dirac field in the Reissner-Nordstrom-de Sitter spacetime, J. High Energy Phys. 01 (2019) 123.

[36] K. Destounis, Charged fermions and strong cosmic censorship, Phys. Lett. B 795, 211 (2019).

[37] M. Rahman, S. Chakraborty, S. SenGupta, and A. A. Sen, Fate of strong cosmic censorship conjecture in presence of higher spacetime dimensions, J. High Energy Phys. 03 (2019) 178.

[38] H. Liu, Z. Tang, K. Destounis, B. Wang, E. Papantonopoulos, and H. Zhang, Strong cosmic censorship in higherdimensional Reissner-Nordstrom-de Sitter spacetime, J. High Energy Phys. 03 (2019) 187.

[39] O. J. C. Dias, H. S. Reall, and J. E. Santos, Strong cosmic censorship: Taking the rough with the smooth, J. High Energy Phys. 10 (2018) 001.

[40] R. Luna, M. Zilhao, V. Cardoso, J. L. Costa, and J. Natario, Strong cosmic censorship: The nonlinear story, Phys. Rev. D 99, 064014 (2019).

[41] O. J. C. Dias, F. C. Eperon, H. S. Reall, and J. E. Santos, Strong cosmic censorship in de Sitter space, Phys. Rev. D 97, 104060 (2018).

[42] B. Gwak, Strong cosmic censorship under quasinormal modes of non-minimally coupled massive scalar field, Eur. Phys. J. C 79, 767 (2019).

[43] H. Guo, H. Liu, X.-M. Kuang, and B. Wang, Strong cosmic censorship in charged de Sitter spacetime with scalar field non-minimally coupled to curvature, Eur. Phys. J. C 79, 891 (2019).

[44] G. W. Gibbons, Aspects of Born-Infeld theory and string/M theory, Rev. Mex. Fis. 49, 19 (2003) [AIP Conf. Proc. 589, 324 (2001)].

[45] T. K. Dey, Born-Infeld black holes in the presence of a cosmological constant, Phys. Lett. B 595, 484 (2004).

[46] R.-G. Cai, D.-W. Pang, and A. Wang, Born-Infeld black holes in (A)dS spaces, Phys. Rev. D 70, 124034 (2004). 
[47] S. Fernando, Thermodynamics of Born-Infeld-anti-de Sitter black holes in the grand canonical ensemble, Phys. Rev. D 74, 104032 (2006).

[48] S. Gunasekaran, R. B. Mann, and D. Kubiznak, Extended phase space thermodynamics for charged and rotating black holes and Born-Infeld vacuum polarization, J. High Energy Phys. 11 (2012) 110.

[49] D.-C. Zou, S.-J. Zhang, and B. Wang, Critical behavior of Born-Infeld AdS black holes in the extended phase space thermodynamics, Phys. Rev. D 89, 044002 (2014).

[50] X.-X. Zeng, X.-M. Liu, and L.-F. Li, Phase structure of the Born-Infeld-anti-de Sitter black holes probed by non-local observables, Eur. Phys. J. C 76, 616 (2016).

[51] P. Wang, H. Wu, and H. Yang, Thermodynamics and phase transitions of nonlinear electrodynamics black holes in an extended phase space, J. Cosmol. Astropart. Phys. 04 (2019) 052.

[52] P. Wang, H. Wu, and H. Yang, Thermodynamics and phase transition of a nonlinear electrodynamics black hole in a cavity, J. High Energy Phys. 07 (2019) 002.

[53] J. Jing and S. Chen, Holographic superconductors in the Born-Infeld electrodynamics, Phys. Lett. B 686, 68 (2010).

[54] M. Baggioli and O. Pujolas, On effective holographic Mott insulators, J. High Energy Phys. 12 (2016) 107.

[55] E. Kiritsis and L. Li, Quantum criticality and DBI magnetoresistance, J. Phys. A 50, 115402 (2017).

[56] Jun Tao, Peng Wang, and Haitang Yang, Testing holographic conjectures of complexity with Born-Infeld black holes, Eur. Phys. J. C 77, 817 (2017).

[57] X. Guo, P. Wang, and H. Yang, Membrane paradigm and holographic DC conductivity for nonlinear electrodynamics, Phys. Rev. D 98, 026021 (2018).

[58] S. Cremonini, A. Hoover, and L. Li, Backreacted DBI magnetotransport with momentum dissipation, J. High Energy Phys. 10 (2017) 133.
[59] P. Wang, H. Wu, and H. Yang, Holographic DC conductivity for backreacted nonlinear electrodynamics with momentum dissipation, Eur. Phys. J. C 79, 6 (2019).

[60] Q. Gan, P. Wang, and H. Yang, Temperature dependence of in-plane resistivity and inverse hall angle in NLED holographic model, Commun. Theor. Phys. 71, 577 (2019).

[61] P. Wang, H. Wu, and H. Yang, Thermodynamics and weak cosmic censorship conjecture in nonlinear electrodynamics black holes via charged particle absorption, arXiv:1904 .12365 .

[62] T.-T. Hu, Y. Song, S. Sun, H.-B. Li, and Y.-Q. Wang, Weak cosmic censorship in Born-Infeld electrodynamics and bound on charge-to-mass ratio, arXiv:1906.00235.

[63] S. Fernando and D. Krug, Charged black hole solutions in Einstein-Born-Infeld gravity with a cosmological constant, Gen. Relativ. Gravit. 35, 129 (2003).

[64] S. Fernando, Born-Infeld-de Sitter gravity: Cold, ultra-cold and Nariai black holes, Int. J. Mod. Phys. D 22, 1350080 (2013).

[65] A. Jansen, Overdamped modes in Schwarzschild-de Sitter and a Mathematica package for the numerical computation of quasinormal modes, Eur. Phys. J. Plus 132, 546 (2017).

[66] A.P. Jansen, Qnmspectral, https://github.com/APJansen/ QNMspectral.

[67] https://centra.tecnico.ulisboa.pt/network/grit/files/ringdown/.

[68] N. Breton, T. Clark, and S. Fernando, Quasinormal modes and absorption cross-sections of Born-Infeld-de Sitter black holes, Int. J. Mod. Phys. D 26, 1750112 (2017).

[69] A. Ishibashi and H. Kodama, Stability of higher dimensional Schwarzschild black holes, Prog. Theor. Phys. 110, 901 (2003). 